\title{
Theory of Lattice and Electronic Fluctuations in Weakly Localized Spin-Peierls Systems
}

\author{
C. Bourbonnais and B. Dumoulin \\ Centre de Recherche en Physique du Solide et Département de Physique, \\ Université de Sherbrooke, Sherbrooke, Québec, Canada J1K 2R1.
}

(July 1996)

\begin{abstract}
A theoretical approach to the influence of one-dimensional lattice fluctuations on electronic properties in weakly localized spin-Peierls systems is proposed using the renormalization group and the functional integral techniques. The interplay between the renormalization group flow of correlated electrons and one-dimensional lattice fluctuations is taken into account by the one-dimensional functional integral method in the adiabatic limit. Calculations of spin-Peierls precursor effects on response functions are carried out explicitely and the prediction for the temperature dependent magnetic susceptibility and nuclear relaxation is compared with available experimental data for $(\mathrm{TMTTF})_{2} \mathrm{PF}_{6}$.
\end{abstract}

\section{INTRODUCTION}

Among the possible structural phase transitions in low-dimensional systems, the spin-Peierls (SP) instability has recently received particular attention. [1.2. In contrast with the Peierls transition, whose normal phase is metallic, pronounced insulating properties and antiferromagnetic spin correlations are found far ahead of the SP ordering temperature. However, owing to the quasi-one-dimensional character of these systems, precursor effects should appear in both types of transitions. Since spin correlations are essentially one-dimensional in character, lattice fluctuations should emerge as diffuse scattering in x-ray experiments, and as a so-called pseudo-gap effect in magnetic properties. The pseudo-gap effect is well known to be the hallmark of strongly anisotropic Peierls systems [1.,3], but surprisingly, its observation is a rather uncommon feature of most SP systems and the underlying explanation for this is not really known so far [2]. Correspondingly, the incitement to achieve a theoretical description of fluctuation effects in the SP case did not receive any experimental justification, so that most theoretical efforts were rather devoted to the description of the ordered state [2, 1 . $(\mathrm{TMTTF})_{2} \mathrm{X}$ and $(\mathrm{BCPTTF})_{2} \mathrm{X}$ with $\mathrm{X}=\mathrm{PF}_{6}, \mathrm{AsF}_{6}$, has prompted a renewed interest for this problem [6 10]. In both cases, one-dimensional SP precursors are clearly seen in structural and magnetic measurements well above the true SP ordering temperature.

This paper will be entirely devoted to the theoretical description of one-dimensional lattice and electronic correlations in SP systems of the (TMTTF $)_{2} \mathrm{X}$ series. Unlike (BCPTTF $)_{2} \mathrm{X}$, which are already good insulators at room temperature, (TMTTF $)_{2} \mathrm{X}$ are weakly localized and still show a metallic behaviour in this temperature range. It is only around $T_{\rho} \simeq 220 \mathrm{~K}$ that carriers undergo a Mott-Hubbard localization, below which the resistivity becomes thermally activated 11]. Albeit the magnetic susceptibility remains unaffected at $T_{\rho}$, NMR spin-lattice relaxation rate measurements have revealed the existence of strong 1D antiferromagnetic spin correlations below this temperature range [7.12]. Below 60K, one-dimensional SP lattice softening is borned out by x-rays and has a marked influence on the temperature dependence of both susceptibility and nuclear relaxation [6.,7]. In order to tackle the problem of one-dimensional electronic and lattice fluctuations effects in SP systems we shall combine the renormalization group technique for fermions and the functional-integral method [9.10]. A similar approach has been recently applied -with some success- to strongly localized SP systems of the $(\mathrm{BCPTTF})_{2} \mathrm{X}$ series [10]. Since the itineracy of carriers is much more pronounced in the high temperature domain of (TMTTF $)_{2} \mathrm{X}$ series, both spin and charge degrees of freedom must be considered in the renormalization flow, which in practice makes the extension of the previous approach slightly more complicated.

\section{THE MODEL AND THE PARTITION FUNCTION}

In setting up the essential ingredients of the model, it is first convenient to study the partition function of the electron-acoustic-phonon interaction model, namely when direct electron-electron interaction is absent. The corresponding hamiltonian is well known to take the form [13]: 


$$
H=\sum_{p, k, \sigma} \epsilon_{p}(k) c_{p, k, \sigma}^{\dagger} c_{p, k, \sigma}+\sum_{Q} \omega(Q)\left(b_{Q}^{\dagger} b_{Q}+\frac{1}{2}\right)+(L)^{-1 / 2} \sum_{\{p, k, Q, \sigma\}} g(k, Q) c_{p, k+Q, \sigma}^{\dagger} c_{-p, k, \sigma}\left(b_{-Q}^{\dagger}+b_{Q}\right),
$$

where $\epsilon_{p}(k)=v_{F}\left(p k-k_{F}\right)$ is the linearized spectrum around the 1D Fermi points $p k_{F}= \pm k_{F}$ for free fermions and $\omega(Q)=\omega_{D}|\sin (Q a / 2)|$ is the dispersion relation for the phonons $(\hbar=1)$. Here $v_{F}$ is the Fermi velocity and $\omega_{D}$ is the Debye frequency, which coincides with the phonon energy at $Q= \pm 2 k_{F}(= \pm \pi / a$, when the band is half-filled). As for the interacting part, the electron-phonon coupling constant is given by:

$$
g(k, Q)=i 4 \frac{g_{a}}{\sqrt{2 M \omega(Q)}} \sin (Q a / 2) \cos (k a+Q a / 2),
$$

where $M$ is the molecular mass and $g_{a}=-d t_{a} / d x>0$ is the spatial modulation of the longitudinal hopping integral $t_{a}$. The dynamics of phonons being purely harmonic, their integration in the partition function $Z=\operatorname{Tr} e^{-\beta H}$ can be carried out exactly, yielding an effective retarded interaction among electrons $\left(\beta=1 / T, k_{B}=1\right)$. The result can be put in the following functional-integral form

$$
Z=\int\left[d \psi^{*}\right][d \psi] e^{S_{\mathrm{e}}^{0}\left[\psi^{*}, \psi\right]} \exp \left(-\int_{0}^{\beta} d \tau_{1} d \tau_{2} \mathcal{H}\left(\tau_{1}-\tau_{2}\right)\right),
$$

over the anticommuting Grassman variables $\psi^{(*)}$. The free electronic action is given by

$$
S_{\mathrm{e}}^{0}\left[\psi^{*}, \psi\right]=\int_{0}^{\beta} d \tau \sum_{p, k, \sigma} \psi_{p, \sigma}^{*}(k, \tau)\left[G_{p}^{0}(k, \tau)\right]^{-1} \psi_{p, \sigma}(k, \tau),
$$

where $G_{p}^{0}(k, \tau)=\left[-\partial / \partial \tau-\epsilon_{p}(k)\right]^{-1}$ is the free electron propagator. As for the retarded interaction $\mathcal{H}\left(\tau_{1}-\tau_{2}\right)$, we will proceed to a "g-ology" decomposition which only retains $2 k_{F}$ phonon exchange between electrons at $\pm k_{F}$, with the result

$$
\begin{gathered}
\mathcal{H}\left(\tau_{1}-\tau_{2}\right)=(2 L)^{-1} \sum_{\{p, k, q, \sigma\}} g_{1, \mathrm{ph}}\left(\tau_{1}-\tau_{2}\right) \psi_{p, \sigma_{1}}^{*}\left(k_{1}+p 2 k_{F}+q, \tau_{1}\right) \psi_{-p, \sigma_{2}}^{*}\left(k_{2}-p 2 k_{F}-q, \tau_{2}\right) \\
\times \psi_{p, \sigma_{2}}\left(k_{2}, \tau_{2}\right) \psi_{-p, \sigma_{1}}\left(k_{1}, \tau_{1}\right) \\
+(2 L)^{-1} \sum_{\{p, k, q, \sigma\}} g_{3, \mathrm{ph}}\left(\tau_{1}-\tau_{2}\right) \\
\begin{array}{l}
\psi_{-p, \sigma_{1}}^{*}\left(k_{1}+p 2 k_{F}-q-p G, \tau_{1}\right) \psi_{-p, \sigma_{2}}^{*}\left(k_{2}-p 2 k_{F}+q, \tau_{2}\right) \\
\times \psi_{p, \sigma_{2}}\left(k_{2}, \tau_{2}\right) \psi_{p, \sigma_{1}}\left(k_{1}, \tau_{1}\right),
\end{array}
\end{gathered}
$$

where $G=4 k_{F}$ is a reciprocal lattice vector. The retarded backscattering and umklapp coupling constants are given by

$$
\begin{aligned}
g_{1, \mathrm{ph}}\left(\tau_{1}-\tau_{2}\right) & =g\left(-p k_{F}, p 2 k_{F}\right) g\left(p k_{F},-p 2 k_{F}\right) D^{0}\left(2 k_{F}, \tau_{1}-\tau_{2}\right) \\
& =\frac{8 g_{a}^{2}}{M \omega_{D}} D^{0}\left(2 k_{F}, \tau_{1}-\tau_{2}\right), \\
g_{3, \mathrm{ph}}\left(\tau_{1}-\tau_{2}\right) & =-\eta g_{1, \mathrm{ph}}\left(\tau_{1}-\tau_{2}\right)
\end{aligned}
$$

where $D^{0}\left(2 k_{F}, \tau_{1}-\tau_{2}\right)=-\left[e^{-\omega_{D}\left|\tau_{1}-\tau_{2}\right|}+2\left(e^{\beta \omega_{D}}-1\right)^{-1} \cosh \left(\omega_{D}\left(\tau_{1}-\tau_{2}\right)\right)\right]$ is the phonon propagator at $2 k_{F}$. Here, $\eta \leq 1$ is a positive constant that reflects the half-filled character of the band. For a single half-filled band, $\eta=1$ while for systems like (TMTTF) $)_{2} \mathrm{X}$ with a quarter-filled band and a small dimerization gap, one can take $\eta \ll 1$ [14]. From above, one observes that, for exchange of $2 k_{F}$ acoustic phonons, $g_{3, \mathrm{ph}}$ and $g_{1, \mathrm{ph}}$ are opposite in sign. The retarded interaction can then be written uniquely in terms of composite fields:

$$
\mathcal{H}\left(\tau_{1}-\tau_{2}\right)=\int d x \sum_{M} g_{\mathrm{ph}}^{M}\left(\tau_{1}-\tau_{2}\right) O^{M *}\left(x, \tau_{1}\right) O^{M}\left(x, \tau_{2}\right),
$$

where we have introduced the composite field

$$
O^{M *}(x, \tau)=\frac{1}{2}\left(O^{*}(x, \tau)+M O(x, \tau)\right),
$$

with

$$
O(x, \tau)=\sum_{\sigma} \psi_{-, \sigma}^{*}(x, \tau) \psi_{+, \sigma}(x, \tau)
$$


These corresponds to "site" $(M=+)$ and "bond" $(M=-)$ charge-density-wave (CDW) correlations at half-filling. The related combinations of couplings are given by

$$
g_{\mathrm{ph}}^{M}\left(\tau_{1}-\tau_{2}\right)=g_{1, \mathrm{ph}}\left(\tau_{1}-\tau_{2}\right)+M g_{3, \mathrm{ph}}\left(\tau_{1}-\tau_{2}\right) .
$$

In order to analyze these correlations, we apply an Hubbard-Stratonovich transformation on the retarded part of the interaction

$$
\begin{aligned}
\left.Z=\int\left[d \phi^{*}\right][d \phi]\left[d \phi^{M}\right] e^{S_{e}^{0}[\psi * \psi \psi}\right] & \exp \left\{-\int d x d \tau_{1} d \tau_{2} \sum_{M= \pm} \phi^{M}\left(x, \tau_{1}\right)\left|g_{\mathrm{ph}}^{M}\left(\tau_{1}-\tau_{2}\right)\right|^{-1} \phi^{M}\left(x, \tau_{2}\right)\right\} \\
& \times \exp \left\{-\int d x d \tau \sum_{M= \pm} \lambda_{M} O^{M}(x, \tau) \phi^{M}(x, \tau)\right\}
\end{aligned}
$$

where $\phi^{ \pm}$are real auxiliary fields for site and bond fluctuations respectively, and $\lambda_{+}=2, \lambda_{-}=2 i$. It is worth noting that, in the absence of umklapp scattering, $g_{\mathrm{ph}}^{-}=g_{\mathrm{ph}}^{+}$and there is no difference between bond and site density wave fluctuations so that both auxiliary fields will combine to produce amplitude and phase fluctuations of the complex field $\phi=\phi^{+}+i \phi^{-}=|\phi| e^{i \varphi}$, as found in incommensurate Peierls systems.

Unretarded repulsive electron-electron interaction at half-filling will promote bond with respect to site electronic correlations. These essential ingredients of the SP instability can be added to the model by following the "g-ology" prescription [15], in which the direct interaction among right- and left-moving carriers is decomposed in terms of backward $\left(g_{1}\right)$, forward $\left(g_{2}\right)$ and $\left(g_{3}\right)$ umklapp scattering coupling constants. The full partition function of the model in the Fourier-Matsubara space then becomes

$$
\begin{aligned}
& Z\left[h^{M}\right]=\int\left[d \psi^{*}\right][d \psi]\left[d \phi^{M}\right] \exp \left\{S_{e}^{0}\left[\psi^{*}, \psi\right]+S^{0}\left[\phi^{M}\right]+S_{\lambda}\left[\psi^{*}, \psi, \phi^{M}\right]+S_{I}\left[\psi^{*}, \psi\right]+S_{h}\left[\psi^{*}, \psi, h^{M}\right]\right\} \\
& \equiv \int\left[d \psi^{*}\right][d \psi]\left[d \phi^{M}\right] \exp \left\{\sum_{p \widetilde{k}, \sigma}\left[G_{p}^{0}(\widetilde{k})\right]^{-1} \psi_{p, \sigma}^{*}(\widetilde{k}) \psi_{p, \sigma}(\widetilde{k})-\sum_{\widetilde{Q}}\left|\phi^{M}(\widetilde{Q})\right|^{2}\left|g^{M}\left(\omega_{m}\right)\right|^{-1}\right. \\
&+ \sqrt{\frac{T}{L}} \sum_{\widetilde{Q}} \lambda_{M} O^{M}(\widetilde{Q}) \phi^{M}(\widetilde{Q}) \\
&+\frac{T}{2 L} \sum_{\{p, \tilde{k}, \tilde{Q}, \sigma\}} g_{1} \psi_{p, \sigma_{1}}^{*}\left(\tilde{k}_{1}+\tilde{Q}\right) \psi_{-p, \sigma_{2}}^{*}\left(\tilde{k}_{2}-\tilde{Q}\right) \psi_{-p, \sigma_{1}}\left(\tilde{k}_{1}\right) \psi_{p, \sigma_{2}}\left(\tilde{k}_{2}\right) \\
&+\frac{T}{2 L} \sum_{\{p, \tilde{k}, \tilde{q}, \sigma\}} g_{2} \psi_{p, \sigma_{1}}^{*}\left(\tilde{k}_{1}+\tilde{q}\right) \psi_{-p, \sigma_{2}}^{*}\left(\tilde{k}_{2}-\tilde{q}\right) \psi_{p, \sigma_{1}}\left(\tilde{k}_{1}\right) \psi_{-p, \sigma_{2}}\left(\tilde{k}_{2}\right) \\
&+\frac{2 T}{L} \sum_{\{p, \tilde{k}, \tilde{Q}, \sigma\}} g_{3} \psi_{p, \sigma_{1}}^{*}\left(\tilde{k}_{1}+\tilde{Q}_{p}\right) \psi_{p, \sigma_{2}}^{*}\left(\tilde{k}_{2}-\tilde{Q}_{p}+p \tilde{G}\right) \psi_{-p, \sigma_{1}}\left(\tilde{k}_{1}\right) \psi_{-p, \sigma_{2}}\left(\tilde{k}_{2}\right) \\
&\left.+\sum_{\widetilde{Q}, \mu}\left(O_{\mu}^{M}(\widetilde{Q}) h_{\mu}^{M *}(\widetilde{Q})+\mathrm{H} . c\right)\right\},
\end{aligned}
$$

where $\left.\widetilde{k}=\left(k, \omega_{n}=(2 n+1) \pi T\right)\right), \widetilde{Q}_{p}=\left(p Q, \omega_{m}=2 \pi m T\right), \widetilde{G}=\left(4 k_{F}, 0\right), G_{p}^{0}(\widetilde{k})=\left[i \omega_{n}-\epsilon_{p}(k)\right]^{-1}$, and $g^{M}\left(\omega_{m}\right)=$ $8 g_{a}^{2}\left(M \omega_{D}\right)^{-1}(1-M \eta) D^{0}\left(2 k_{F}, \omega_{m}\right)$. The integration measures are $\left[d \psi^{*}\right][d \psi]=\prod_{p \widetilde{k}, \sigma} d \psi_{p, \sigma}^{*}(\widetilde{k}) d \psi_{p, \sigma}(\widetilde{k})$ and $\left[d \phi^{M}\right]=$ $\prod_{M, \widetilde{Q}>0}\left|g_{\mathrm{ph}}^{M}\left(\omega_{m}\right)\right|^{-1} d \phi^{M}(\widetilde{Q}) d \phi^{M}(-\widetilde{Q})$ for fermion and auxiliary fields, respectively. Here

$$
D^{0}\left(2 k_{F}, \omega_{m}\right)=\frac{-2 \omega_{D}}{\omega_{m}^{2}+\omega_{D}^{2}}
$$

corresponds to the bare phonon propagator. Finally, $S_{h}$ is an additional term which couples an infinitesimal source field $h_{\mu}^{M}$ to site bond $(M= \pm, \mu=0) \mathrm{CDW}$ and site $(M=+, \mu=1,2,3) \mathrm{SDW}$ correlations. These correspond to composite fields defined by $O_{\mu}^{M *}(\widetilde{Q})=\frac{1}{2}\left(O_{\mu}^{*}(\widetilde{Q})+M O_{\mu}(\widetilde{Q})\right)$, with $O_{\mu=0,1,2,3}^{*}(\widetilde{Q})=(T / L)^{1 / 2} \sum_{\widetilde{k}, \alpha, \beta} \psi_{+, \alpha}^{*}(\widetilde{k}+\widetilde{Q}) \sigma_{\mu}^{\alpha \beta} \psi_{-, \beta}(\widetilde{k})$, $\sigma_{1,2,3}$ and $\sigma_{0}$ being the Pauli and the identity matrices respectively. In the following the source-field term will be useful for the calculation of relevant response functions for the SP instability. 


\section{RENORMALIZATION GROUP RESULTS}

The problem of low-energy electronic and lattice correlations reduces to the study of interacting electrons coupled to the fluctuating field $\phi^{M}$, which can be done by first applying the renormalization group approach developped in Ref. [16,10] for the fermion degrees of freedom. It consists in first integrating high-energy fermion states, namely we write $\left.\psi^{*}\right) \rightarrow \psi^{(*)}+\bar{\psi}^{(*)}$, where $\bar{\psi}^{(*)}$ describes degrees of freedom to be integrated over in the outer energy shell of tickness $\frac{1}{2} E_{0}(\ell) d \ell$ on both sides of the Fermi level at $\pm k_{F}$ and for all $\omega_{n}$. Here $E_{0}(\ell)=E_{0} e^{-\ell}$ is the band energy cut-off at the step $\ell$ and $E_{0} \equiv 2 E_{F}$ is the initial band width, which is twice the Fermi energy. Keeping the $\phi^{M}$ 's fixed, this is formally written as

$$
\begin{aligned}
Z\left[h^{M}\right] & \propto \int_{<}\left[d \psi^{*}\right][d \psi]\left[d \phi^{M}\right] e^{S\left[\psi^{*}, \psi, \phi^{M}, h^{M}\right]_{\ell}-\beta \mathcal{F}\left[\phi^{\mathcal{M}}\right]_{\ell}} \int_{o . s}\left[d \bar{\psi}^{*}\right][d \bar{\psi}] e^{\bar{S}_{e}^{0}\left[\bar{\psi}^{*} \bar{\psi}\right]}\left(e^{\bar{S}_{\lambda}+\bar{S}_{I}+\bar{S}_{h}}\right) \\
& =\int_{<}\left[d \psi^{*}\right][d \psi]\left[d \phi^{M}\right] e^{S\left[\psi^{*}, \psi, \phi^{M}, h^{M}\right]_{\ell}-\beta \mathcal{F}\left[\phi^{\mathcal{M}}\right]_{\ell}} \exp \left(\sum_{n} \frac{1}{n !}\left\langle\left(\bar{S}_{\lambda}+\bar{S}_{I}+\bar{S}_{h}\right)^{n}\right\rangle_{o . s}\right) \\
& \propto \int_{<}\left[d \psi^{*}\right][d \psi]\left[d \phi^{M}\right] e^{S\left[\psi^{*}, \psi, \phi^{M}, h^{M}\right]_{\ell+d \ell}-\beta \mathcal{F}\left[\phi^{M}\right]_{\ell+d \ell}},
\end{aligned}
$$

which leads to a recursion relation for electronic parameters of the action including those related to source fields for the calculation of response function (see below) and for $\mathcal{F}\left[\phi^{M}\right]_{\ell}$, which is a free-energy functional which collects all the contributions in the auxilliary field $\phi^{M}$. At finite temperature, the partial integration is conducted down to $\ell_{T}=\ln \left(E_{F} / T\right)$. In the present renormalization group procedure, electronic degrees of freedom are treated in the continuum limit so that the discreteness of the underlying lattice will be neglected in the following [10].

\section{A. Electronic part}

\section{Couplings and one-particle self-energy}

It is useful for the following discussion to recall the well-known two-loop RG results for the purely electronic part $\left(\phi^{M}=0\right)$ in zero field $\left(h^{M}=0\right)$. The effect of the lattice fluctuations and the calculation of relevant response functions will be considered afterwards. In this purely electronic limit, the single-particle fermion propagator transforms according to the recursion relation $z_{1}(\ell+d \ell)\left[G_{p}^{0}\right]^{-1}=z_{1}(\ell) z_{1}(d \ell)\left[G_{p}^{0}\right]^{-1}$. At two-loop level the outer-shell correction to $z_{1}(d \ell)$ comes from the $n=2\left\langle\bar{S}_{\mathrm{I}}^{2}\right\rangle_{\text {o.s }}$ terms of $(10)$, which yields the flow equation:

$$
\frac{d \ln z_{1}^{-1}}{d \ell}=-\frac{1}{16}\left\{\left[2 \widetilde{g}_{2}(\ell)-\widetilde{g}_{1}(\ell)\right]^{2}-\widetilde{g}_{3}^{2}(\ell)+3\left[\widetilde{g}_{1}^{2}+3 \widetilde{g}_{3}^{2}\right]\right\}
$$

where the $\widetilde{g}_{i}(\ell) \equiv g_{i}(\ell)\left(\pi v_{F}\right)^{-1}$ are the normalized coupling constants at $\ell$. The latter transform as $\widetilde{g}_{i}(\ell+d \ell)=$ $\widetilde{g}_{i}(\ell) z_{1}^{-2}(d \ell) z_{2,3,4}(d \ell)$, which, together with $z_{1}$, are obtained from one-loop $\left\langle\bar{S}_{\mathrm{I}}^{2}\right\rangle_{o . s}$ and two-loop $\left\langle\bar{S}_{\mathrm{I}}^{3}\right\rangle_{o . s}$ outer shell corrections to two-particle four-point vertex functions $\Gamma_{1,2,3}(\ell+d \ell)=z_{2,3,4}(d \ell) \Gamma_{1,2,3}(\ell)$. This is known to yield the flow equations [17,15,16]

$$
\begin{aligned}
\frac{d \widetilde{g}_{1}}{d \ell} & =-\widetilde{g}_{1}^{2}-\frac{1}{2} \widetilde{g}_{1}^{3}, \\
\frac{d\left(2 \widetilde{g}_{2}-\widetilde{g}_{1}\right)}{d \ell} & =\widetilde{g}_{3}^{2}\left[1-\frac{1}{2}\left(2 \widetilde{g}_{2}-\widetilde{g}_{1}\right)\right], \\
\frac{d \widetilde{g}_{3}}{d \ell} & =\widetilde{g}_{3}\left(2 \widetilde{g}_{2}-\widetilde{g}_{1}\right)\left[1-\frac{1}{4}\left(2 \widetilde{g}_{2}-\widetilde{g}_{1}\right)\right]-\frac{1}{4} \widetilde{g}_{3}^{3},
\end{aligned}
$$

For $(\mathrm{TMTTF})_{2} \mathrm{X}$ compounds, $\widetilde{g}_{1} \simeq \widetilde{g}_{2}>0$ are repulsive at $\ell=0$, whereas a finite dimerization of the TMTTF stacks can be parametrized by a small and positive $\widetilde{g}_{3} \ll \widetilde{g}_{1}$ 14.97. One then gets the inequality $\widetilde{g}_{1}-2 \widetilde{g}_{2}<\widetilde{g}_{3}$ indicating that umklapp scattering is relevant. The flow of charge couplings $2 \widetilde{g}_{2}-\widetilde{g}_{1}$ and $\widetilde{g}_{3}$ then scales towards strong coupling while the spin coupling $\widetilde{g}_{1}$ is found to be marginally irrelevant with the fixed point values $\widetilde{g}_{3}^{*}(\ell \rightarrow \infty) \rightarrow 2, \widetilde{g}_{2}^{*}(\ell \rightarrow \infty) \rightarrow 1$, and $\widetilde{g}_{1}^{*} \rightarrow 0$. The strong coupling sector of the RG flow is reached when $g_{3}\left(\ell \equiv \ell_{\rho}\right) \approx 1$, where $\ell_{\rho}=\ln \left(E_{F} / T_{\rho}\right)$ defines the temperature scale for the presence of a charge (Mott-Hubbard) gap $\Delta_{\rho} \equiv 2 T \rho$. Neglecting transients between weak and strong coupling regimes, one-particle self-energy corrections resulting from the solution of Eq. (11) can be put in the following scaling form 


$$
z_{1}^{-1}(\ell) \approx z_{1}^{-1}\left(\ell_{\rho}\right)\left(E_{0}(\ell) / \Delta_{\rho}\right)^{\theta_{\rho}^{*}}
$$

which depicts the reduction of the quasi-particle weight at the Fermi level with $\theta^{*}\left(g_{1}^{*}, g_{2}^{*}, g_{3}^{*}\right)=3 / 4$ being evaluated at the fixed point.

\section{Response functions}

The pertinent electronic response functions involved in the description of the SP instability in one dimension are those related to site $\operatorname{SDW}(\mu \neq 0, M=+)$ and bond $\operatorname{CDW}(\mu=0, M=-)$ correlations $[9]$. They can be readily calculated via the renormalization of the source field term $S_{h}$ [16] which, at scale $\ell$, reads

$$
S_{h}\left[\psi^{*}, \psi, h^{M}\right]_{\ell}=\sum_{\{\mu, M, \widetilde{Q}\}}\left\{z_{\mu}^{M}(\ell) h_{\mu}^{M *}(\widetilde{Q}) O_{\mu}^{M}(\widetilde{Q})+\text { H.c. }-\chi_{\mu}^{M}\left(2 k_{F}, \ell\right) h_{\mu}^{M *}(\widetilde{Q}) h_{\mu}^{M}(\widetilde{Q})\right\}
$$

where $z_{\mu}^{M}(\ell)$ is the renormalization factor for the pair vertex part, while

$$
\chi_{\mu}^{M}\left(2 k_{F}, \ell\right)=-\left(\pi v_{F}\right)^{-1} \int_{0}^{\ell} \bar{\chi}_{\mu}^{M}\left(\ell^{\prime}\right) d \ell^{\prime},
$$

is the $2 k_{F}$ response function and $\bar{\chi}_{\mu}^{M}=\left(z_{\mu}^{M}\right)^{2}$ is the auxiliary susceptibility in the $(\mu, M)$ channel considered . The one- and two-loop corrections to $z_{\mu}^{M}$ come from the outer shell averages $\left\langle\bar{S}_{h} \bar{S}_{I}\right\rangle_{\text {o.s }}$ and $\left\langle\bar{S}_{h} \bar{S}_{I}^{2}\right\rangle_{\mathrm{o} . \mathrm{s}}$ for $n=2$ and $n=3$ respectively. This leads to the flow equation

$$
\frac{d \ln \bar{\chi}_{\mu}^{M}}{d \ell}=\widetilde{g}_{\mu}^{M}(\ell)-\frac{1}{2}\left\{\widetilde{g}_{1}^{2}(\ell)+\widetilde{g}_{2}^{2}(\ell)-\widetilde{g}_{1}(\ell) \widetilde{g}_{2}(\ell)+\frac{1}{2} \widetilde{g}_{3}^{2}(\ell)\right\},
$$

where $\widetilde{g}^{ \pm}(\ell)=\widetilde{g}_{2}(\ell) \mp \widetilde{g}_{3}(\ell)-2 \widetilde{g}_{1}(\ell)$ for the CDW channel and $\widetilde{g}_{\mu \neq 0}^{+}(\ell)=\widetilde{g}_{2}(\ell)+\widetilde{g}_{3}(\ell)$ for the SDW channel. Following the example of (13) for the one-particle self-energy renormalization, $\bar{\chi}_{\mu}^{M}$ can be expressed in the following scaling form

$$
\bar{\chi}_{\mu}^{M}(\ell) \approx \bar{\chi}_{\mu}^{M}\left(\ell_{\rho}\right)\left(E_{0}(\ell) / \Delta_{\rho}\right)^{-\gamma_{\mu}^{* M}}
$$

where $\bar{\chi}_{\mu}^{M}\left(\ell_{\rho}\right)$ is the weak coupling contribution below the charge gap. The exponents $\gamma_{1,2,3}^{*+}=\gamma^{*-}=3 / 2$ obtained by evaluating the r.h.s of (16) at the fixed point indicate that only site SDW and bond CDW correlations are singular at half-filling. It should be mentionned, however, the $g_{i}^{*}$ obtained at two-loop level are well known to overestimate the values of the exponents. Higher order contributions are expected to bring them closer to the exact value

$$
\gamma_{1,2,3}^{*+}=\gamma^{*-} \equiv \gamma^{*}=1
$$

which is known to result from more elaborate calculations at $\ell \gg \ell_{\rho}$ [18.

Before closing this section, it is useful for applications (see $\S$ III.B.5 and $\S$ IV.B) to compute the imaginary part of the retarded response function at low (real) frequency. From Ref. [16], it is directly connected to $\chi_{\mu}^{M}$ as follows:

$$
\operatorname{Im} \chi_{\mu}^{M}\left(2 k_{F}+q, \omega\right)=\bar{\chi}_{\mu}^{M}(T) \operatorname{Im} \chi^{0}\left(q+2 k_{F}, \omega\right), \quad \omega \rightarrow 0
$$

for $v_{F} q \ll T$, where

$$
\begin{aligned}
\operatorname{Im} \chi^{0}\left(q+2 k_{F}, \omega\right) & =\frac{2 T}{L} \operatorname{Im} \sum_{k, \omega_{n}} G_{-}^{0}\left(k, \omega_{n}\right) G_{+}^{0}\left(k+2 k_{F}+q, \omega_{n}+\omega+i 0^{+}\right) \\
& =-\frac{8 \omega}{T v_{F} \cosh ^{2}\left(\beta v_{F} q / 4\right)}, \quad \omega \rightarrow 0,
\end{aligned}
$$

is the imaginary part of the low-frequency non-interacting response function near $2 k_{F}$ (see Appendix A.2). 


\section{B. Influence of lattice fluctuations}

\section{Ginzburg-Landau functional}

When the lattice auxiliary field $\phi^{M}$ is taken into account, the partial integration (8) generates a series of terms in $n \geq 2$ powers of the $\phi^{M}$ 's via the closed fermion loops $\left\langle\bar{S}_{\lambda}^{n}\right\rangle / n$ !. This will not only lead to corrections for $S^{0}\left[\phi^{M}\right]_{\ell}$ for $n=2$, but it yields a recursion relation for the quantum Ginzburg-Landau free-energy functional $\mathcal{F}\left[\phi^{M}\right]_{\ell}$, when combined to the $n>2$ terms [10,16]. Since only $2 k_{F}$ bond correlations are singular in the CDW channel for repulsive couplings, only the dependence on the bond field $\phi^{-}$needs to be retained, with the result

$$
\beta \mathcal{F}\left[\phi^{-}\right]_{\ell+d \ell}=\beta \mathcal{F}\left[\phi^{-}\right]_{\ell}-\sum_{n \geq 2} \sum_{\widetilde{Q}} B_{n}(d \ell) \phi^{-}\left(\widetilde{Q}_{1}\right) \ldots \phi^{-}\left(\widetilde{Q}_{n}\right) \delta_{\Sigma_{\tilde{Q}}=\widetilde{G}} .
$$

The quadratic $(n=2)$ and quartic $(n=4)$ outer shell contributions lead to

$$
\begin{aligned}
& B_{2}(d \ell)=\left(\pi v_{F}\right)^{-1}\left[z^{-}(\ell)\right]^{2} d \ell, \\
& B_{4}(d \ell) \simeq-\frac{T}{L} \frac{7 \zeta(3)\left[z^{-}(\ell)\right]^{4}}{2 \pi^{3} v_{F}\left[E_{0}(\ell)\right]^{2}} d \ell,
\end{aligned}
$$

where $\zeta(3) \simeq 1.2 \ldots$. For the problem at hand, one can assume that adiabaticity between electrons and the lattice is sufficiently strong that quantum lattice corrections can be neglected, thereby allowing the static limit. Up to the quartic term, one obtains the following Ginzburg-Landau free energy functional (in real space)

$$
\mathcal{F}\left[\phi^{-}\right]_{\ell_{T}}=\int d x\left[a(T)\left(\phi^{-}(x)\right)^{2}+c\left(\frac{d \phi^{-}}{d x}\right)^{2}+b\left(\phi^{-}(x)\right)^{4}\right]
$$

at temperature $T$, where $a(T), c$ and $b$ are the Ginzburg-Landau parameters. The coefficients $a(T)$ and $c$ of the quadratic term are respectively given by

$$
\begin{aligned}
a(T) & =\left[g_{\mathrm{ph}}^{-}(0)\right]^{-1}+\chi^{-}\left(2 k_{F}, T\right) \\
& \approx a^{\prime}\left(T / T_{S P}^{0}-1\right) \\
c & \approx \frac{1}{2} a^{\prime}\left(v_{F} / \pi T_{S P}^{0}\right)^{2},
\end{aligned}
$$

where $a^{\prime}=\left(\pi v_{F}\right)^{-1} \bar{\chi}^{-}\left(T_{S P}^{0}\right)$. Here the bond CDW response function $\chi^{-}\left(2 k_{F}, T\right)$ in Eq. (15), together with (17), have been used in the linearization of $a(T)$ around the SP mean-field temperature

$$
T_{S P}^{0} \approx T_{\rho}\left(\left|\widetilde{g}_{\mathrm{ph}}^{-}\right| \bar{\chi}^{-}\left(T_{\rho}\right)\right)^{1 / \gamma^{*}} .
$$

for $T_{\rho} \gg T_{S P}^{0}$. It is worth noting that since $\left|g_{p h}^{-}\right| \propto g_{a}^{2}$, then $T_{S P}^{0} \propto g_{a}^{2}$, which turns out to be the same power dependence on the electron-phonon coupling constant that was found in previous mean-field calculations on more localized systems 河. As for the rigidity parameter $c$, it is readily obtained in Fourier space from the $Q=2 k_{F}+q$ expansion of $\chi^{-}\left(Q, T_{S P}^{0}\right)$ using the approximate expression

$$
\ell\left(T_{S P}^{0}, 2 k_{F}+q\right)=\ln \left\{\frac{E_{F}}{T_{S P}^{0}}\left[1+\left(v_{F} q / \pi T_{S P}^{0}\right)^{2}\right]^{-\frac{1}{2}}\right\},
$$

as boundary condition of the logarithmic integration in (15) near $T_{S P}^{0}$. This form essentially coincides with the $Q$ dependence of the elementary Peierls bubble near $2 k_{F}$. Finally, the flow of the mode-mode coupling in (22) is stopped at $\ell_{T_{S P}^{0}}$ and reads

$$
b \approx\left(\pi v_{F}\right)^{-1} \frac{7 \zeta(3)}{16\left(1+\gamma^{*}\right)\left(\pi T_{S P}^{0}\right)^{2}}\left[\bar{\chi}^{-}\left(T_{S P}^{0}\right)\right]^{2}
$$

for $T_{\rho} \gg T_{S P}^{0}$.

Fluctuation effects in $\phi^{-}$below $T_{S P}^{0}$ are then governed by the classical functional integral $\mathcal{Z}=$ $\int\left[d \phi^{-}\right] \exp \left(-\beta \mathcal{F}\left[\phi^{-}\right]\right)$, which can be carried out exactly using the transfer matrix method [21]. An important quantity to compute is the static 1D SP response function $\chi_{S P}$. From the results of Ref. 21], one finds 


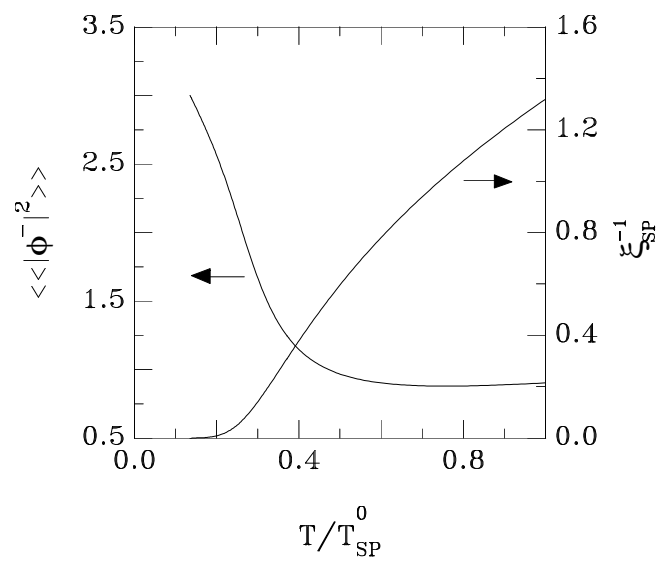

FIG. 1. Temperature variation of the mean square of the SP order parameter (left scale ) and the inverse of the correlation length (right scale) normalized by the lattice constant.

$$
\begin{aligned}
\chi_{S P}\left(2 k_{F}+q, T\right) & =\frac{1}{T} \int d x\left\langle\left\langle\phi^{-}(x) \phi^{-}(0)\right\rangle\right\rangle e^{-i\left(q+2 k_{F}\right) x} \\
& =\frac{2 \beta\left\langle\left\langle\left(\phi^{-}\right)^{2}\right\rangle\right\rangle \xi_{S P}}{1+q^{2} \xi_{S P}^{2}}
\end{aligned}
$$

where

$$
\langle\langle(\ldots)\rangle\rangle=\mathcal{Z}^{-1} \int\left[d \phi^{-}\right](\ldots) \exp \left(-\beta \mathcal{F}\left[\phi^{-}\right]\right)
$$

denotes a statistical average over $\phi^{-}$. Here $\xi_{S P}$ is the correlation length of the real order field $\phi^{-}$, which grows exponentially below $T_{S P}^{0}$ according to

$$
\xi_{S P}=\frac{T}{\rho} \sqrt{\frac{c}{|a|}} e^{\rho / T}
$$

where

$$
\rho=\frac{(2 c)^{\frac{1}{2}}|a|^{\frac{3}{2}}}{b} \approx 3.8 T_{S P}^{0}
$$

for $T \ll T_{S P}^{0}$. The temperature profile of both $\xi_{S P}$ and the mean square fluctuations $\left\langle\left\langle\left(\phi^{-}\right)^{2}\right\rangle\right\rangle$ are plotted in Figure 1 .

\section{Pseudo-gap effect}

The influence of the static SP lattice fluctuations governed by (23) on one-particle electronic properties at the step $\ell_{T}$ of the RG procedure will be analyzed by considering to leading order the one-electron self-energy contribution of Figure 2 [3, which can be obtained from (8) after an integration over the $\phi^{-}$field. The result is

$$
\Sigma_{p}^{*}\left(\widetilde{k},\left\{\phi^{-}\right\}\right)=-T L^{-1} z_{1}^{-1} z_{0}^{-} \sum_{q} G_{-p}^{0}\left(k-p 2 k_{F}-q, i \omega_{n}\right) \chi_{S P}\left(2 k_{F}+q, T\right) .
$$

The bare propagator appearing in $S_{\mathrm{e}}^{0}$ then becomes

$$
\begin{aligned}
{\left[G_{p}\left(\widetilde{k},\left\{\phi^{-}\right\}\right)\right]^{-1} } & =z_{1}\left[G_{p}^{0}(\widetilde{k})\right]^{-1}-\Sigma_{p}^{*}\left(\widetilde{k},\left\{\phi^{-}\right\}\right) \\
& =z_{1}\left(i \omega_{n}-\epsilon_{p}(k)\right)-\frac{z_{1}^{-1}\left(z_{0}^{-}\right)^{2}\left\langle\left\langle\left|\phi^{-}\right|^{2}\right\rangle\right\rangle}{i \omega_{n}+\epsilon_{p}(k)+i v_{F} \xi_{S P}^{-1}(T)} .
\end{aligned}
$$

A relevant quantity to compute is the reduction of the density of states per spin resulting from SP fluctuations 


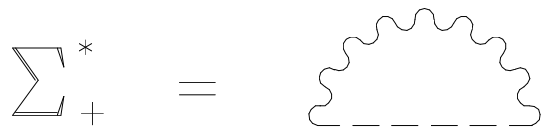

FIG. 2. Leading one-particle self-energy correction due to SP fluctuations. for electrons at $+k_{F}$. The wiggly (dashed) line corresponds to the lattice field $\left(-k_{F}\right)$ propagator $\chi_{S P}$.

a)

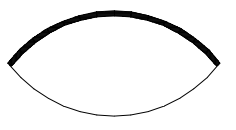

b)
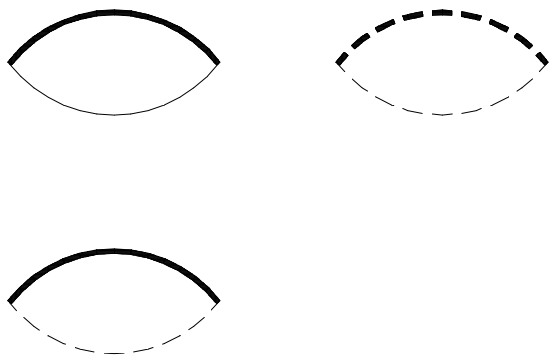

FIG. 3. Dressed electron-hole bubbles in the a) Landau and b) Peierls (or Cooper) channels. Thick and thin continous (dashed) lines correspond to dressed and bare electron propagators, respectively at $+k_{F}\left(-k_{F}\right)$.

(pseudo-gap effect). After analytic continuation of $\left[G_{p}\right]^{-1}$ to real frequencies, this is found to be

$$
\begin{aligned}
D\left[\omega,\left\{\phi^{-}\right\}\right] & =-\frac{z_{1}}{\pi L} \sum_{p, k} \operatorname{Im} G_{p}\left(k, \omega,\left\{\phi^{-}\right\}\right) \\
& =\frac{1}{\pi v_{F}} \frac{\alpha\left[2(\vartheta+\kappa]^{1 / 2}\right.}{\left[2(\vartheta+\kappa)-\alpha^{2}\right] \vartheta}
\end{aligned}
$$

where the presence of the renormalization factor $z_{1}$ ensures that the pseudo-gap effect is the result of lattice fluctuations. Following the notation of Ref. [3], we have

$$
\begin{aligned}
\alpha & =v_{F} \xi_{S P}^{-1}\left\langle\left\langle\left|\Phi^{-}\right|^{2}\right\rangle\right\rangle^{-1 / 2} \\
\tilde{\omega} & =\omega\left\langle\left\langle\left|\Phi^{-}\right|^{2}\right\rangle\right\rangle^{-1 / 2} \\
\kappa & =1+\frac{1}{4} \alpha^{2}-\tilde{\omega}^{2} \\
\vartheta & =\left(\kappa^{2}+\tilde{\omega}^{2} \alpha^{2}\right)^{1 / 2}
\end{aligned}
$$

where we have defined $\left|\Phi^{-}\right|^{2}=z_{1}^{-2}\left(z^{-}\right)^{2}\left|\phi^{-}\right|^{2}$. Therefore, as the temperature decreases below the characteristic $T_{S P}^{0}$, lattice fluctuations grow and will progressively freeze electronic degrees of freedom. In turn, this reduction will affect the RG flow of various quantities considered in §III. The inclusion of lattice fluctuation effects in the RG flow will be considered to lowest order where vertex corrections due to exchange of lattice fluctuations are neglected. For example, at one-loop level of the RG, this amounts to substituting $G\left(\widetilde{k},\left\{\phi^{-}\right\}\right)$for one of the propagators appearing in the formal expression of the Landau, Peierls, and Cooper elementary susceptibilities (Figure 3 and Appendix A). At the two-loop level, all the relevant next-to-leading singular diagrams shown in Figure 4 involve absorption and emission of an electron-hole pair at small $\widetilde{q}$ in the intermediate state, which can be dressed by lattice fluctuations (Appendix B). From the results of Appendix A and B, this amounts to replacing the outer shell logarithmic contribution of all diagrams by the following expression

$$
d \ell \rightarrow \pi v_{F} D\left[E_{0}(\ell) / 2,\left\{\phi^{-}\right\}\right] d \ell
$$

where $D\left[E_{0}(\ell) / 2,\left\{\phi^{-}\right\}\right]$is density of states (32) in the presence of a pseudo-gap.

Substituting this RG generator in the flow equation for the renormalization factor $z_{1}^{-1}$ in (11) will slow down the electronic contribution to the decay of the quasi-particle weight below $T_{S P}^{0}$. Following the example of the purely electronic case, the approximate "scaling" (transient-free) form (13) then becomes

$$
z_{1}^{-1}(T) \approx z_{1}^{-1}\left(T_{S P}^{0}\right)\left(T / T_{S P}^{0}\right)^{\theta_{\rho}^{*}(T)}
$$

where $z_{1}^{-1}\left(T_{S P}^{0}\right)$ is given by (11) and $\theta_{\rho}^{*}(T)=\pi v_{F} D\left[T,\left\{\phi^{-}\right\}\right] \theta_{\rho}^{*}$ becomes a temperature dependent exponent below $T_{S P}^{0}$. 
a)
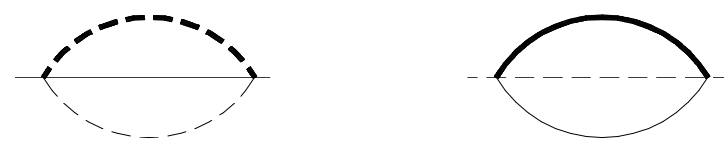

b)
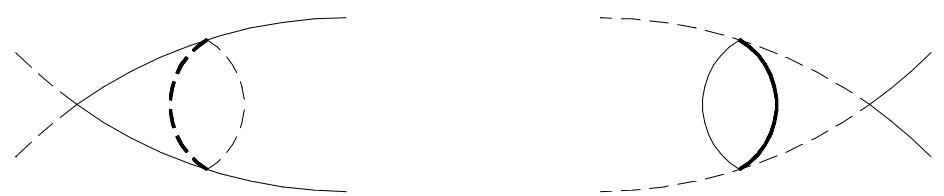

FIG. 4. Two-loop a) one-particle self-energy and b) four points vertex singular diagrams involved in the RG flow including the leading order corrections due to lattice fluctuations.

\section{Staggered response functions}

The pseudo-gap will affect electronic correlations of the $(\mu, M)$ channel as well. Indeed, substituting (34) in (16) yields the following approximate "scaling" form

$$
\bar{\chi}_{\mu}^{M}\left(T,\left\{\phi^{-}\right\}\right) \approx \bar{\chi}_{\mu}^{M}\left(T_{S P}^{0}\right)\left(T / T_{S P}^{0}\right)^{-\gamma^{*}(T)},
$$

where $\bar{\chi}_{\mu}^{M}\left(T_{S P}^{0}\right)$ is given by (17) and $\gamma^{*}(T)=\pi v_{F} D\left[T,\left\{\phi^{-}\right\}\right] \gamma^{*}$.

As for the imaginary part (19), the above results and Appendix A.2 lead to

$$
\begin{aligned}
\operatorname{Im} \chi_{\mu}^{M}\left(q+2 k_{F}, \omega,\left\{\phi^{-}\right\}\right) & =-\bar{\chi}_{\mu}^{M}\left(T,\left\{\phi^{-}\right\}\right) \operatorname{Im} \chi\left(q+2 k_{F}, \omega,\left\{\phi^{-}\right\}\right) \\
& \simeq-\bar{\chi}_{\mu}^{M}\left(T,\left\{\phi^{-}\right\}\right) \frac{\pi D\left[v_{F} q / 2,\left\{\phi^{-}\right\}\right]}{8 T \cosh ^{2}\left(\beta v_{F} q / 4\right)} \omega, \quad \omega \rightarrow 0
\end{aligned}
$$

which is further reduced by lattice fluctuations.

\section{Magnetic susceptibility}

The calculation of the spin response function $\chi_{s}$ at small $(q, \omega)$ is known in the purely electronic situation [19]. Its generalization when lattice fluctuations are present is straightforward and yields

$$
\chi_{s}\left(q, \omega,\left\{\phi^{-}\right\}\right)=\frac{2 \mu_{B}^{2} \chi\left(q, \omega,\left\{\phi^{-}\right\}\right)}{1-\frac{1}{2} g_{1}(T) \chi\left(q, \omega,\left\{\phi^{-}\right\}\right)},
$$

where in the above scheme of approximation $\chi\left(q, \omega,\left\{\phi^{-}\right\}\right)$is the elementary electron-hole bubble dressed by lattice fluctuations (Fig. 3a and Appendix A.1), and $g_{1}(T)$ is given by (12) at $\ell_{T}$. According to the results of Appendix A, one finds

$$
\begin{aligned}
& \operatorname{Re} \chi\left(q, \omega,\left\{\phi^{-}\right\}\right)=\int_{0}^{T} D\left[\omega^{\prime},\left\{\phi^{-}\right\}\right]\left(-\frac{\delta n}{\delta \omega^{\prime}}\right) d \omega^{\prime} \sum_{p} \frac{p v_{F} q}{p v_{F} q-\omega}, \\
& \operatorname{Im} \chi\left(q, \omega,\left\{\phi^{-}\right\}\right)=\int_{0}^{T} D\left[\omega^{\prime},\left\{\phi^{-}\right\}\right]\left(-\frac{\delta n}{\delta \omega^{\prime}}\right) d \omega^{\prime} \sum_{p} p \pi v_{F} q \delta\left(p v_{F} q-\omega\right),
\end{aligned}
$$

for the real and imaginary parts of the dressed electron-hole bubble. It follows that in the static $(\omega \rightarrow 0)$ and uniform $(q \rightarrow 0)$ limit, the temperature variation of the spin susceptibility $\chi_{s}\left(T,\left\{\phi^{-}\right\}\right)$will be depressed in the presence of lattice fluctuations that grow up below $T_{S P}^{0}$ (Fig. 5.a ). In the very low-temperature domain, the SP correlation length becomes exponentially large and the magnetic susceptibility becomes thermally activated. When these temperature conditions prevail, the system is almost long-range ordered. 


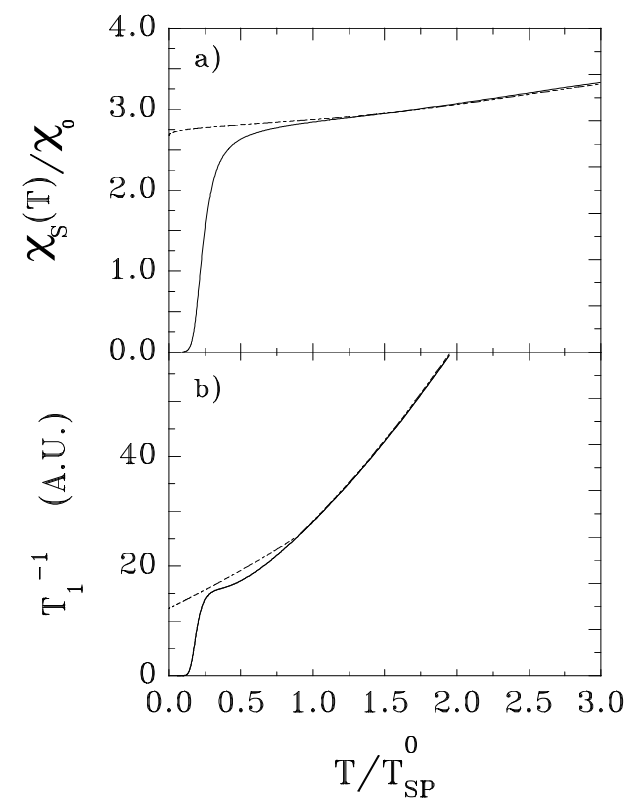

FIG. 5. Calculated low temperature variation of a) the uniform magnetic susceptibility and b) nuclear relaxation rate in the presence (continuous line) and absence (dashed line) of lattice fluctuations.

\section{Nuclear relaxation rate}

From the above results, the temperature dependence of the nuclear spin-lattice relaxation rate $T_{1}^{-1}$ can be readily calculated. The basic expression for $T_{1}^{-1}$ in one dimension is well known to be

$$
T_{1}^{-1}=|\bar{A}|^{2} T \int d Q \frac{\operatorname{Im} \chi(Q, \omega)}{\omega},
$$

which can be taken in the limit $\omega \rightarrow 0$. Here $\operatorname{Im} \chi$ is the imaginary part of the retarded spin response function and $\bar{A}$ is a constant proportional to the hyperfine coupling [20]. It is well established that uniform $(Q \sim 0)$ and $\operatorname{AF}\left(Q \sim 2 k_{F}\right)$ spin fluctuations give the essential contributions to the relaxation in one dimension [19], allowing in turn to make the following decomposition

$$
T_{1}^{-1}=T_{1}^{-1}[Q \sim 0]+T_{1}^{-1}\left[Q \sim 2 k_{F}\right] .
$$

For the staggered part $T_{1}^{-1}\left[Q \sim 2 k_{F}\right]$, we have seen from (37) that $\operatorname{Im} \chi\left(2 k_{F}+q, \omega\right)$ ( whose definition coincides with $\left.-\operatorname{Im} \chi_{\mu \neq 0}^{M}\left(2 k_{F}+q, \omega\right)\right)$ is peaked when $q$ lies in the interval $\left[-T / v_{F}, T / v_{F}\right]$, which leads to

$$
T_{1}^{-1}\left[Q \sim 2 k_{F}\right] \simeq C_{1} T D\left[T,\left\{\phi^{-}\right\}\right] \bar{\chi}_{\mu}^{+}\left(T,\left\{\phi^{-}\right\}\right),
$$

where $C_{1}=\pi v_{F}^{-1}|\bar{A}|^{2} \tanh (1 / 4)$.

As for the uniform contribution, the use of (38-39) immediately leads to

$$
T_{1}^{-1}[Q \sim 0] \simeq C_{0} T \frac{D\left[T,\left\{\phi^{-}\right\}\right]}{\left[1-\frac{1}{2} g_{1}(T) D\left[T,\left\{\phi^{-}\right\}\right]\right]^{2}},
$$

where $C_{0}=4 \pi\left(v_{F}\right)^{-1}|\bar{A}|^{2}$.

As expected, the reduction of spin fluctuations by short range lattice correlations below $T_{S P}^{0}$ will decrease the amplitude of the relaxation via the reduction of the density of states. At sufficiently low temperature, namely when the correlation length $\xi_{S P}$ becomes exponentially large, one finds $T_{1}^{-1} \sim \chi_{s} \sim e^{-\rho T_{S P}^{0} / T}$. In the high-temperature regime, lattice fluctuations are small and the uniform component $T_{1}^{-1} \sim C_{0} T \chi_{s}^{2}$ eventually dominates the relaxation. Using the scaling form (36) in (42) and (43), the temperature dependence of $T_{1}^{-1}$ is summarized in Figure 5.b where it is compared to the case without fluctuations. 


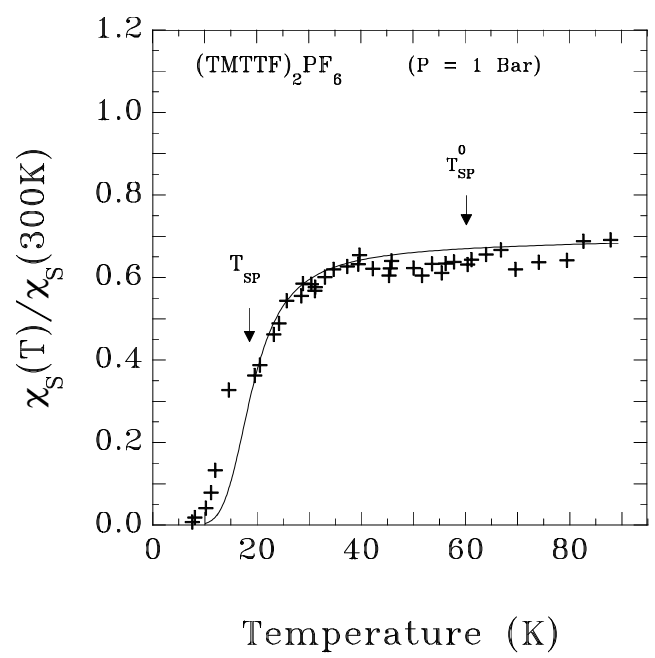

FIG. 6. Comparison between calculated (continuous line) and observed (crosses) temperature profiles of magnetic susceptibility for (TMTTF $)_{2} \mathrm{PF}_{6}$. The data are taken from Ref.[7].

\section{APPLICATION TO THE SP SYSTEM (TMTTF) $)_{2} \mathrm{PF}_{6}$}

By way of application of the present theory to the sulphur based organic compound (TMTTF $)_{2} \mathrm{PF}_{6}$. Previous analysis of this system in the normal phase using the one-dimensional electron gas model can be used for the determination of the input bare parameters of the purely electronic part of the model. NMR analysis of Wzietek et al., [12] have shown that $\widetilde{g}_{1} \simeq \widetilde{g}_{2} \simeq .9$, with $E_{F} \simeq 1600 \mathrm{~K}$, give a rather good description of the temperature dependent magnetic susceptibility in the high temperature domain of this material. As for the observed characteristic temperature scale $T_{\rho} \approx 220 \mathrm{~K}$ (below which the system presents insulating properties), it can be used to identify, together with (12), the low-temperature domain of strong umklapp scattering, which then allows to take $\widetilde{g}_{3} \approx .2[9]$. As for the input parameters for the lattice component, one will fix the value of $T_{S P}^{0}$ at $60 \mathrm{~K}$ which is the characteristic temperature scale for the onset of strong lattice fluctuations in x-ray experiments [6]. From the above set of figures all quantities of interest can be calculated.

\section{A. Magnetic susceptibility}

The temperature-dependent EPR spin susceptibility (TMTTF) ${ }_{2} \mathrm{PF}_{6}$ measured by Creuzet et al., 7 is reported in the low-temperature domain in Figure $6 . \chi_{s}(T)$ decreases monotonously from the high temperature domain and becomes weakly temperature dependent near $80 \mathrm{~K}$, which is typical of all members of the sulphur series in the normal state. However, in the low temperature domain below $60 \mathrm{~K}$, the spin susceptibility decreases by roughly $40 \%$ down to the true SP transition at $T_{S P} \approx 19 \mathrm{~K}$, below which it becomes thermally activated. Using the above set of parameters for the model, the theoretical prediction for $\chi_{s}\left(T,\left\{\phi^{-}\right\}\right)$is illustrated in Figure 6 and gives a fairly good description of the temperature variation of $\chi_{s}$ in the SP pseudo-gap regime. At very low temperature, the present theory predicts a thermally activated behaviour when $\xi_{S P}$ grows exponentially, which is found to mimic the actual temperature dependence below the true transition temperature at $19 \mathrm{~K}$. However, a more realistic description of the SP system in this low temperature region, would require the inclusion of the interchain coupling.

\section{B. Nuclear Relaxation}

The temperature profile of $T_{1}^{-1}$ for $(\mathrm{TMTTF})_{2} \mathrm{PF}_{6}$, measured by Creuzet et al., [7] is given in Figure 7. From the analysis of nuclear relaxation in the high temperature domain $T_{S P}^{0}<T<T_{\rho}$, where SP fluctuations are weak, the contribution to $T_{1}^{-1}$ is well known to be purely electronic in character. A quantitative description of the relaxation rate in this regime can be obtained from (42) and (43) neglecting the dependence on $\phi^{-}$[12]. Below $T_{S P}^{0}$, the relaxation rate shows a $30 \%$ decrease between $T_{S P}^{0}$ and $T_{S P}$ due to one-dimensional lattice fluctuations. According to (42) and (42) both the staggered and uniform parts of the relaxation are affected below $T_{S P}^{0}$. Thus, using these expressions 


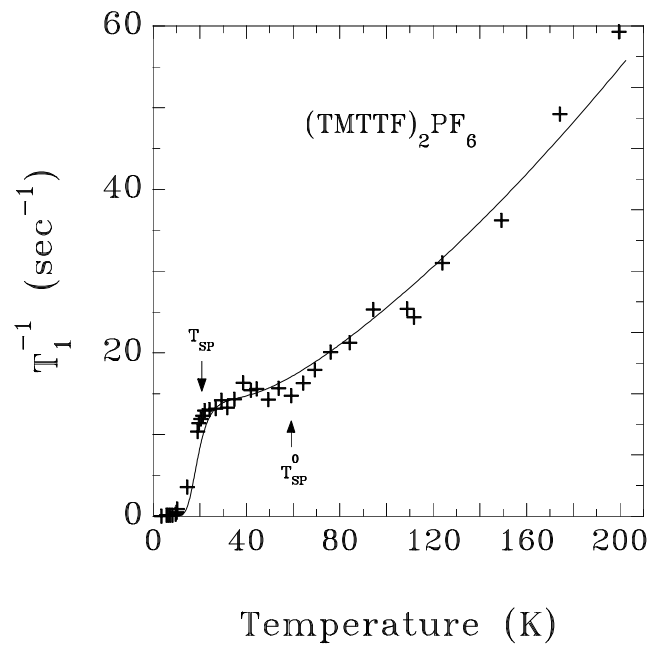

FIG. 7. Comparison between calculated (continuous line) and observed (crosses) temperature profiles of nuclear relaxation rate for (TMTTF $)_{2} \mathrm{PF}_{6}$. The data are taken from Ref.[7].

for the above set of parameters, one obtains the $T_{1}^{-1}$ temperature profile shown in Figure 7 . The theoretical curve is obtained from the expressions (43), (42) and (36) in which the values of the constants $C_{0}\left(\pi v_{F}\right)^{-1} \simeq 12.1$ and $C_{1}\left(\pi v_{F}\right)^{-1} T_{\rho} \bar{\chi}_{\mu}^{+}\left(T_{\rho}\right) \simeq 9.6$ results from the analysis of $T_{1}^{-1}$ data made by Wzietek al., [12] in the high temperature domain $T \gg T_{S P}^{0}$.

\section{ACKNOWLEDGMENTS}

The authors thank J.-P. Pouget and L. G. Caron for numerous discussions. We would also like to thank D. Senechal for useful comments about the manuscript. Financial support from the Natural Sciences and Engineering Research Council of Canada (NSERC), le Fonds pour la Formation de Chercheurs et l'Aide à la Recherche du Gouvernement du Québec (FCAR) and Canadian Institute for Advanced Research (CIAR) is gratefully acknowledged.

\section{APPENDIX A: ELEMENTARY SUSCEPTIBILITIES IN PRESENCE OF PHONONS}

In this appendix, we proceed to the calculation of the elementary electron-hole bubbles dressed by lattice fluctuations in the Landau, Peierls and Cooper channels, respectively.

\section{Susceptibility at small $q$ and $\omega$}

The expression corresponding to the electron-hole bubble of Figure 3a is

$$
\chi\left(\widetilde{q},\left\{\phi^{-}\right\}\right)=-\frac{T}{L} \sum_{p, \widetilde{k}} G_{p}\left(\widetilde{k},\left\{\phi^{-}\right\}\right) G_{p}^{0}(\widetilde{k}+\widetilde{q}),
$$

for one spin orientation. Using the spectral representation of $G_{p}\left(\widetilde{k},\left\{\phi^{-}\right\}\right)$and performing the fermion frequency sum, one finds

$$
\chi\left(\widetilde{q},\left\{\phi^{-}\right\}\right)=-(\pi L)^{-1} \sum_{p, k} \int_{-\infty}^{+\infty} d \omega^{\prime} \operatorname{Im} G_{p}\left(k, \omega^{\prime},\left\{\phi^{-}\right\}\right) \frac{n\left[\epsilon_{p}(k+q)\right]-n\left[\omega^{\prime}\right]}{i \omega_{m}+\epsilon_{p}(k+q)-\omega^{\prime}} .
$$

Since most of the spectral weight appears in the region $\omega^{\prime} \approx \epsilon_{p}(k)$, one can replace $\epsilon_{p}(k)$ by $\omega^{\prime}$ in the ratio of the above integral, which can be cut off at $\pm E_{0} / 2$. From the definition of the density of states (32), one finds, after analytic continuation to real frequencies, 


$$
\begin{aligned}
& \operatorname{Re} \chi\left(q, \omega,\left\{\phi^{-}\right\}\right)=\frac{1}{2} \int_{-E_{0} / 2}^{+E_{0} / 2} D\left[\omega^{\prime},\left\{\phi^{-}\right\}\right]\left(-\frac{\delta n}{\delta \omega^{\prime}}\right) d \omega^{\prime} \sum_{p} \frac{p v_{F} q}{p v_{F} q-\omega} \\
& \operatorname{Im} \chi\left(q, \omega,\left\{\phi^{-}\right\}\right)=\frac{1}{2} \int_{-E_{0} / 2}^{+E_{0} / 2} D\left[\omega^{\prime},\left\{\phi^{-}\right\}\right]\left(-\frac{\delta n}{\delta \omega^{\prime}}\right) d \omega^{\prime} \sum_{p} p \pi v_{F} q \delta\left(p v_{F} q-\omega\right) .
\end{aligned}
$$

Here $D\left(\omega,\left\{\phi^{-}\right\}\right)$is the density of states per spin in the presence of the spin-Peierls pseudo-gap. The calculation of $\chi\left(\widetilde{q},\left\{\phi^{-}\right\}\right)$at the step $\ell$ of the renormalization group procedure gives the same expression, except for $E_{0}$ which is replaced by $E_{0}(\ell)$.

\section{Peierls and Cooper susceptibilities}

The calculation of the Peierls electron-hole bubble of Figure 3b starts with the following expression

$$
\chi\left(2 k_{F}+q, \omega_{m},\left\{\phi^{-}\right\}\right)=\frac{2 T}{L} \sum_{k, \omega_{n}} G_{-}\left(k, \omega_{n},\left\{\phi^{-}\right\}\right) G_{+}^{0}\left(k+2 k_{F}+q, \omega_{n}+\omega_{m}\right),
$$

for both spin orientations. At zero external variables and after a summation over the fermion frequencies and the use of the spectral representation for $G_{-}\left(\widetilde{k},\left\{\phi^{-}\right\}\right)$, one gets

$$
\chi\left(2 k_{F},\left\{\phi^{-}\right\}\right)=-\frac{2}{\pi L} \sum_{k} \int_{-\infty}^{+\infty} d \omega^{\prime} \operatorname{Im} G_{-}\left(k, \omega,\left\{\phi^{-}\right\}\right) \frac{n\left[\omega^{\prime}\right]-n\left[-\epsilon_{-}(k)\right]}{\omega^{\prime}+\epsilon_{-}(k)},
$$

which actually coincides with the real part of the Peierls bubble. Assuming that $\operatorname{Im} G_{-}\left(k, \omega^{\prime},\left\{\phi^{-}\right\}\right)$is peaked in the region $\omega^{\prime} \approx \epsilon_{-}(k)$, from which one replaces $\epsilon_{-}(k)$ by $\omega^{\prime}$ in the ratio appearing in the integral, we find

$$
\chi\left(2 k_{F},\left\{\phi^{-}\right\}\right)=-\int_{0}^{E_{0} / 2} D\left[\omega^{\prime},\left\{\phi^{-}\right\}\right] d \omega^{\prime} \frac{\tanh \left(\beta \omega^{\prime} / 2\right)}{\omega^{\prime}} .
$$

As for the electron-electron (Cooper) elementary bubble at zero external variables corresponding to the expression

$$
\chi\left(\left\{\phi^{-}\right\}\right)=\frac{2 T}{L} \sum_{\widetilde{k}} G_{-}\left(\widetilde{k},\left\{\phi^{-}\right\}\right) G_{+}^{0}(-\widetilde{k}),
$$

the property $G_{+}^{0}\left(-k,-\omega_{n}\right)=-G_{+}^{0}\left(k+2 k_{F}, \omega_{n}\right)$ leads to the relation $\chi\left(2 k_{F},\left\{\phi^{-}\right\}\right)=-\chi\left(\left\{\phi^{-}\right\}\right)$.

Within the renormalization group scheme at $\ell$, the same expressions become

$$
\begin{aligned}
d \chi\left(2 k_{F},\left\{\phi^{-}\right\}\right) & =-D\left[E_{0}(\ell) / 2,\left\{\phi^{-}\right\}\right] d \ell \\
& =-d \chi\left(\left\{\phi^{-}\right\}\right),
\end{aligned}
$$

when evaluated in the outer energy shell. Finally, after analytic continuation to real frequencies of (A4), we can carry over the same type of calculation for the imagnary part of the Peierls bubble at $T$ in the limit of small frequency and we find

$$
\begin{aligned}
\operatorname{Im} \chi\left(2 k_{F}+q, \omega \rightarrow 0,\left\{\phi^{-}\right\}\right) & \simeq-\frac{\pi}{2} \int_{-E_{0} / 2}^{+E_{0} / 2} d \omega^{\prime} D\left[\omega^{\prime},\left\{\phi^{-}\right\}\right]\left(n\left[\omega^{\prime}\right]-n\left[-\omega^{\prime}+v_{F} q\right]\right) \delta\left(\omega^{\prime}+\left(\omega-v_{F} q\right) / 2\right) \\
& =-\frac{\pi D\left[v_{F} q / 2,\left\{\phi^{-}\right\}\right]}{8 T \cosh ^{2}\left(\beta v_{F} q / 4\right)} \omega .
\end{aligned}
$$

\section{APPENDIX B: ONE-PARTICLE SELF-ENERGY AND FOUR-POINT VERTEX PART IN PRESENCE OF PHONONS}

\section{One-particle self-energy}

The expression for the one particle self-energy diagram of Figure 4a reads 


$$
\begin{aligned}
\Sigma_{+}\left(\widetilde{k},\left\{\phi^{-}\right\}\right) & =-2 g^{2} \frac{T^{2}}{L^{2}} \sum_{\widetilde{k^{\prime}}, \widetilde{q}} G_{-}\left(\widetilde{k}^{\prime},\left\{\phi^{-}\right\}\right) G_{-}^{0}\left(\widetilde{k}^{\prime}+\widetilde{q}\right) G_{+}^{0}(\widetilde{k}-\widetilde{q}) \\
& \left.\simeq 2 g^{2} \frac{T}{\pi L^{2}} \sum_{k^{\prime}} \sum_{\widetilde{q}} \int_{-\infty}^{+\infty} d \omega^{\prime} \operatorname{Im} G^{(} k^{\prime}, \omega^{\prime},\left\{\phi^{-}\right\}\right) \frac{n\left[\omega^{\prime}\right]-n\left[\epsilon_{-}\left(k^{\prime}\right)-v_{F} q\right]}{\left[i \omega_{n}-\epsilon_{+}(k)-i \omega_{m}+v_{F} q\right]\left[\omega^{\prime}+i \omega_{m}-\epsilon_{-}\left(k^{\prime}\right)+v_{F} q\right]}
\end{aligned}
$$

where the second line results from a fermion frequency summation and the use of the spectral weight representation. The approximation scheme of Appendix A then allows to put $\epsilon_{-}\left(k^{\prime}\right) \approx \omega^{\prime}$ in the ratio appearing in r.h.s of this last expression and to cut off the integral over $\omega^{\prime}$ at $\pm E_{0} / 2$. In the RG procedure, the outer energy shell evaluation of $\Sigma_{+}$at $\ell$, which is obtained after the frequency sum over $\omega_{m}$ leads to $d \Sigma_{+}=d \Sigma_{+}^{+}+d \Sigma_{+}^{-}$, where

$$
\begin{aligned}
d \Sigma_{+}^{ \pm}\left(\widetilde{k},\left\{\phi^{-}\right\}\right)= & -\frac{g^{2}}{4 \pi} D\left[E_{0}(\ell) / 2,\left\{\phi^{-}\right\}\right] d E_{0}(\ell) \\
& \times \int d q \frac{\left(n\left[ \pm E_{0}(\ell) / 2-v_{F} q\right]-n\left[ \pm E_{0}(\ell) / 2\right]\right)\left(n_{B}\left[-v_{F} q\right]+n\left[v_{F} q+\epsilon_{+}(k)\right]\right)}{2 v_{F} q+i \omega_{n}-\epsilon_{+}(k)} \\
\simeq & -\frac{g^{2}}{8 \pi v_{F}}\left[G^{0}(\widetilde{k})\right]^{-1} D\left[E_{0}(\ell) / 2,\left\{\phi^{-}\right\}\right] d \ell,
\end{aligned}
$$

to leading order in $\left[G^{0}(\widetilde{k})\right]^{-1}=i \omega_{n}-\epsilon_{+}(k)$, for $\beta E_{0}(\ell) \gg 1$, and where $n_{B}[x]=\left(e^{\beta x}-1\right)^{-1}$. Here the integration over the momentum transfer $q$ is found to contribute only in the interval $2 v_{F} q_{0}>2 v_{F}|q|>E_{0}(\ell)$, where $q_{0}$ is a momentum transfer cut-off.

\section{Vertex part}

The evaluation of the two-loop vertex part in the presence of lattice fluctuations proceeds along similar lines. The diagrams of Figure $4 \mathrm{~b}$ corresponds to $\Gamma_{a}^{(2)}+\Gamma_{b}^{(2)}$ and to the generic expression

$$
\Gamma_{a, b}^{(2)}\left(\left\{\phi^{-}\right\}\right)=-g^{2} \frac{T^{2}}{L^{2}} \sum_{\widetilde{k}^{\prime}, \widetilde{q}^{\prime}} G_{\mp}\left(\widetilde{k}^{\prime}, \phi^{-}\right) G_{\mp}^{0}\left(\widetilde{k}^{\prime}-\widetilde{q}^{\prime}\right) G_{ \pm}^{0}\left(\widetilde{k}_{1,2}+\widetilde{q}^{\prime}\right) G_{ \pm}^{0}\left(\widetilde{k}_{1,2}+\widetilde{q}^{\prime}-\widetilde{q}\right) .
$$

In the RG sense one can drop the dependence on the external variables $\left\{\widetilde{k}_{1}, \widetilde{k}_{2}, \widetilde{q}\right\}$. Performing frequency sums on $\omega_{n^{\prime}}$ and $\omega_{m^{\prime}}$ and using the above approximation scheme, one gets

$$
\begin{aligned}
d \Gamma_{a, b}^{(2)} \simeq & g^{2} \frac{1}{32 \pi v_{F}^{2}} D\left[E_{0}(\ell) / 2,\left\{\phi^{-}\right\}\right] d E_{0}(\ell) \\
& \times \int \frac{d q^{\prime}}{q^{\prime 2}}\left(\left[n\left[E_{0}(\ell) / 2+v_{F} q^{\prime}\right]-n\left[E_{0}(\ell) / 2\right]\left[n_{B}\left[-v_{F} q^{\prime}\right]+n\left[v_{F} q^{\prime}\right]\right]+E_{0}(\ell) \rightarrow-E_{0}(\ell)\right)\right. \\
\simeq & g^{2} \frac{1}{8 \pi v_{F}} D\left[E_{0}(\ell) / 2,\left\{\phi^{-}\right\}\right] d \ell,
\end{aligned}
$$

for $2 v_{F} q_{0}>2 v_{F}\left|q^{\prime}\right|>E_{0}(\ell), \beta v_{F} q^{\prime} \gg 1$, and $\beta E_{0}(\ell) \gg 1$.

[1] I. F. Shchegolev, Physica Status Solidi A 12 (1972) 9; G. A. Toombs, Phys. Rep.40 (1978) 181.

[2] J. W. Bray, L. V. Interrante, I. S. Jacobs and J.C. Bonner, in Extended Linear Chain Compounds, edited by J.S. Miller (Plenum, New York, 1983), Vol. 3, P.353

[3] P.A. Lee, T. M. Rice, and P.W. Anderson, Phys. Rev. Lett. 31 (1973) 462.

[4] M. C. Cross and D.S. Fisher, Phys. rev. B 19, (1979) 402.

[5] H. Fukuyama, Synthetic Metals 19 (1987) 63 and references therein.

[6] J. P. Pouget, R. Moret, R. Comes, K. Bechgaard, J. M. Fabre, and L. Giral, Mol. Cryst. Liq. Cryst. 79 (1982) 129.

[7] F. Creuzet, C. Bourbonnais, L. G. Caron, D. Jerome and K. Bechgaard, Synthetic Metals 19 (1987) 299.

[8] Q. Liu, S. Ravy, J.P Pouget, C. Coulon, and C. Bourbonnais, Synthetic Metals, 55-57 (1993) 1840. 
[9] L.G. Caron, C. Bourbonnais, F. Creuzet, and D. Jerome, Synthetic Metals 19 (1987) 69.

[10] B.Dumoulin, C.Bourbonnais, S. Ravy, J.-P. Pouget and C. Coulon Phys. Rev. Lett.76, (1996)1360.

[11] R. Laversanne, C. Coulon, B. Gallois, J.-P. Pouget and R. Moret, J. Physique Lett. (Paris) 45 (1984) L393.

[12] P. Wzietek et al., J. Physique (France), I 3 (1993) 173.

[13] W. D. Su, J. R. Schrieffer and A. J. Heeger, Phys. Rev. B22, (1980) 2099; S. Barisic, Phys. Rev. B 5 (1972) 932.

[14] S. Barisic and S. Brazovskii, in: Recent Developments in Condensed Matter Physics Vol.1, ed. J.T. Devreese (Plenum, New York,1981)p. 237.

[15] See for example, J. Solyom, Adv. Phys. 28 (1979) 201 and references therein.

[16] C. Bourbonnais and L.G. Caron, Int. Journ. Mod. Phys. 5 (1991) 1033; C.Bourbonnais and L.G. Caron J. Physique (Paris) 50 (1989) 2751.

[17] M. Kimura, Prog. Theor. Phys. 53 (1975) 955; I.E. Dzyaloshinskii and A.I. Larkin, Sov. Phys. JETP 34 (1972) 422.

[18] J. Voit, Rep. Prog. Phys.58 (1995) 977 and references therein.

[19] C. Bourbonnais, J.Physique (France) I 3 (1993) 143.

[20] T. Moryia, J. Phys. Soc. Jpn 18, (1974), 2911

[21] W. Dieterich, Adv. Phys. 25, (1976) 615; D. J. Scalapino, M. Sears and R. A. Ferrell, B6, 3409 (1972). 